\title{
Prediction of No Tropic Properties of Novel Drug Modafiendz using in-Silico Method
}

\author{
Kumud Pant, Abhishek Semwal, Devvret Verma, Promila Sharma, Akansha Pal, Neetu Sharma, Akshara \\ Pande, Somya Sinha, Neema Tufchi
}

\begin{abstract}
The development and approval of new drug is a tedious, expensive and highly time-consuming task. The demand of new drugs is increasing, and the development of natural drugs and traditional drugs are re-emerging as a new strategic task. The in-silico techniques have boosted the development of potential drug candidates. One important category of drugs is nootropes. It improves the cognitive function, memory, motivation and creativity in healthy individuals. The demand of the nootropic drugs has skyrocketed in past few decades. Modafiendz is a novel drug that is often used by the consumers as it is having similarity in structure and property with modafinil (Nootropic drug). But no major studies have been carried out on this molecule, so remains an investigatory molecule.

There are several in-silico techniques that can be used to predict the likeness, metabolic activity and pharmacological property of a molecule. In the current study, realizing the importance of modafiendz, various properties of modafiendz have been predicted like metabolism site, ADME properties, metabolite prediction and DNA adduct formation tendencies. The properties of modafiendz were found to be similar to modafinil in various regards of drug likeability, bioavailability, blood brain permeability (BBB), GI absorption and site of metabolism (SOM). The study suggests modafiendz as a better nootropic drug candidate, when compared to modafinil.
\end{abstract}

Keywords: no tropics, modafinil, modafiendz, in-silico analysis, ADME, DNA adduct

When compared to past decades the sales and availability of drugs has increased by many folds. Thiscan be credited to increased research in finding new or improved drugs to treat a specific condition and rapid improvements in industrial processes of drug manufacturing.

Revised Manuscript Received on September 25, 2019.

*Corresponding Author

Kumud Pant, Department of Biotechnology, Graphic Era (Deemed to be University), Dehradun, Uttarakhand, India. E-mail: pant.kumud@gmail.com

Abhishek Semwal, Department of Biotechnology, Graphic Era (Deemed to be University), Dehradun, Uttarakhand, India. E-mail: abhisheksemwal42@gmail.com

Devvret Verma*, Department of Biotechnology, Graphic Era (Deemed to be University), Dehradun, Uttarakhand, India. E-mail: devvret@gmail.com

Promila Sharma, Department of Life Sciences, Graphic Era (Deemed to be University), Dehradun, Uttarakhand, India. E-mail: promilanaut@yahoo.com

Akansha Pal, Department of Biotechnology, Graphic Era (Deemed to be University), Dehradun, Uttarakhand, India. E-mail: akankshapal50@gmail.com

Neetu Sharma, Department of Chemistry, Graphic Era (Deemed to be University), Dehradun, Uttarakhand, India.

Akshara Pande, Department of Computer Science \& Engineering, Graphic Era Hill University, Dehradun, Uttarakhand, India. E-mail: pandeakshara@gmail.com

Somya Sinha, Department of Biotechnology, Graphic Era (Deemed to be University), Dehradun, Uttarakhand, India. Email: somyasinha.bio@geu.ac.in

Neema Tufchi, Department of Biotechnology, Graphic Era (Deemed to be University), Dehradun, Uttarakhand, India. E-mail: neematufchi@gmail.com

\section{INTRODUCTION}

Based on their action and chemical structure the existing drugs can be categorized as antibiotics, antipyretics, analgesics, nootropics and many more [1]. Nootropic drugs also known as smart drugs are one such category that enhances the cognitive performance and work by increasing a number of mental functions such as memory, creativity, attention and motivation [1]. The term 'nootropes' is derived from Greek words 'noos' meaning mind and 'tropein' meaning towards [2]. It is estimated that the demand for nootropics would more than double in 2020 when compared to the demand in 2012 [3]. Some of the popular nootropic drugs include modafinil, adrafinil, GABA (gammaAminobutyric acid) and aniracetam.

The demand for new drugs has exponentially increased in today's era because continuous and excessive use of these medicines has resulted in development of drug resistance. The drugs which were previously good enough to be used in case of a certain diseases are now no longer equally effective and so the search for potent drugs and their analogues are on the rise. However, the task of discovering a new drug and bringing it to the markets in itself is a tedious process. In United States, it takes about 12 to 7 years for a drug to come to market after the beginning of initial trials [4]. Also, there are a number of diseases for treating which no drugs have yet been approved or research is underway. Modafinil is a synthetic CNS (Central Nervous System) stimulant, cognitive enhancer and has alertness promoting property and appears to increase the amount of dopamine outside cells [5]. It is sold in markets as provigil and is prescribed in conditions like narcolepsy and obtrusive sleep apnea [6], [7].It was discovered by chemists at L. Lafon drug company in 1976 while studying about the properties of adrafinil, another drug that was discovered by the same company in $1974[8]$.

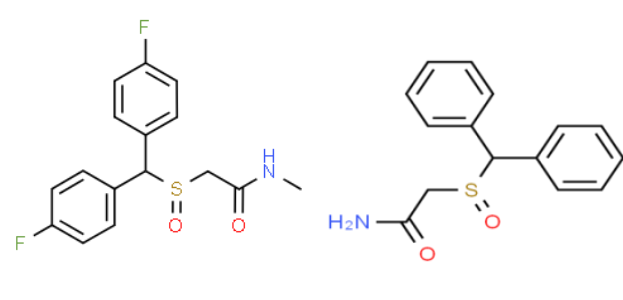

Modafiendz

Modafinil

Fig. 1. - Structures of modafiendz and modafinil

Modafiendz is an allotrope of modafinil shown in figure 1 [7]. Because of the structural similarity of modafiendz to modafinil, it is used by the consumers as a nootropic drug, however its metabolic activity and modesof action are still unknown.

Since modafiendz is a structural analogue of modafinil which is one of the highly used nootropics in history,

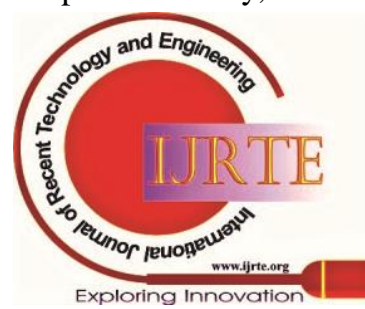




\section{Prediction of No Tropic Properties of Novel Drug Modafiendz using in-Silico Method}

some argue that the former has high potential to replace other nootropics.But this requires further research to conclude whether modafiendz is effective nootropic or not and can it be used to treat other medical conditions as well.

While looking for new potential drugs, one of the major steps is to find out the metabolic pathway of the drug. Theknowledge about metabolic pathways plays an important role in understanding the internal functioning of an organism. It is by using this knowledge that alteration of metabolic pathways can be done to carry out or enhance the production of a desired product from microorganisms. There is a wide array of software and online resources for predicting the metabolic pathway and other properties of a new molecule. These online resources help in boosting the research output by furnishing important scientific information accessible to all.

One of the first steps in metabolic pathway identification involves finding the sites of metabolism (SOM) in the given molecule, finding potential metabolites and checking for their ADME properties.

While carrying out reconstruction of metabolic pathway of an organism a draft of all the known metabolic reactions taking place, needs to be considered along with knowledge of other parameters. The computational methods of metabolic pathway prediction involve matching a given query or molecule with the database of reaction rules for various enzymes (according to knownlaws of chemistry and mathematics). Following the similar strategy, efforts were made for finding relationship between the rates of reaction of enzymes on substrate molecules, later it could explain the dynamic functions of pathways such as glycolysis [9].

The majorenzymes involved in drug metabolism are CYP450, CYb5 besides others, falling under the category of monooxygenases [10]. Cytochrome P450(CYP450) family of enzymes are heme proteins with heme as the cofactor [11]. They carry out important function ofmetabolism of xenobiotics and drugs in mammals and are found in vast variety of life forms [12]. This family of enzymes is responsible for nearly $75 \%$ of the drug metabolism and 5 of the CYP450 enzymes present in humans are involved in nearly $95 \%$ of the reactions [13]. Mainly present in the liver they are also found in other organs like small intestines, kidney and the gut wall [14]. It is because of the important role of CYP450 enzymes in drug metabolism in humans that it is primarily considered in designing software and online resources for predicting the binding probabilities of the major CYP enzymes on sites in a given molecule.

The other important analysis is prediction of DNA adduct formation property of the drug. Drugs are bioactivated by drug-metabolizing enzymes into reactive metabolites, which then conjugate to sites in proteins or DNA to form adducts [15]. DNA adducts can be used as a significant biomarker for exposure to carcinogenic agents. It is because of the property of DNA adduct formation being related to carcinogenicity of a molecule it can be deduced that which molecules are reactive with DNA [16].

As an amalgamation of various strategies of drug metabolism and pathway prediction (as stated above), in this paper an attempt has been made to unravel thesame for modafiendz by using various publicly available software and servers. The prediction of DNA adducts formation tendencies of the metabolites of modafinil and modafiends was also carried out to check for any carcinogenic nature. Lastly comparison of modafiendz with modafinil has also been carried out to check for difference in their properties and also validating the accuracy and reliability of in-slico predictions. The process flow chart is shown in figure 2 .

\section{METHODS}

\begin{tabular}{|c|}
\hline \multicolumn{1}{|c|}{ Selection of Compounds } \\
\hline Checking for SOM using SMARTCyp and SOMP \\
\hline \\
\hline Predicting ADME properties using SwissADME \\
\hline Predicting probable metabolites using BioTransformer \\
\hline \\
\hline Checking for DNA adduct formation tendencies of metabolites \\
using xenosite reactivity 2.0 \\
\hline
\end{tabular}

Fig. 2. Process flowchart of the study

\section{A. Site of metabolism prediction}

\section{1) SMARTCyp}

SMARTCyp is one of the most widely used online resources for the prediction of the site of metabolism in a given molecule. It was introduced in 2010. It uses the 2D structure of the molecule in .sdf, .mol or SMILES (simplified molecular input line entry system) format and it predicts the CYP (CYP3A4, CYP2C9 and CYP2D6) mediated metabolism sites. Prediction from SMARTCyp is based on the density functional theory (DFT) to compute the oxidation states of the aliphatic carbons [17]-[19]. SMARTCyp is a free to access online resource. It works well for SOM prediction for smaller molecules.

\section{2) SOMP (Site Of Metabolism Prediction)}

SOMP is a free online resource for prediction of SOM of CYP2D6, CYP3A4, CYP2C19, CYP2C9, CYP1A2 and UGT. It was introduced in 2015. It is based on PASS(Prediction of activity spectra for substances) and LMNA descriptors, the PASS algorithm carries out the estimation of the likely profiles of biological activity based on the input structural formula [20].

\section{B. ADME (absorption, distribution, metabolism, excretion) properties - SwissADME}

SwissADME is a free web tool that predicts the ADME(absorption, distribution, metabolism and excretion) properties and the drug likeliness of the molecule [21]. For predicting the ADME properties of the molecule, the input file can be imported from the local library, can be drawn by using structure editor tool or by simply providing the SMILES format. It is maintained by Swiss Institute of Bioinformatics(SIB). It also checks the molecule for a number of rules of drug likeness like Lipinski's rule of 5, Ghose's rule, Veber's rule, Egan's rule and Muegge's rule [22]-[26].

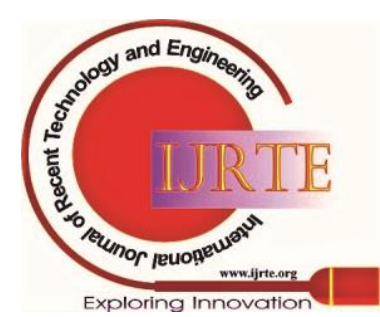




\section{Metabolite analysis - BioTransformer}

BioTransformer is a free online tool that carries out the prediction of metabolism of small molecules in mammals [14]. It was launched in 2018 and this project was supported by Canadian Institute of Health Research (CIHR). BioTransformer combines knowledge centric and a machine learning based methods to predict metabolism in a molecule, it is capable of predicting metabolic reactions in human gut environment and also in environmental microbial setting [14].

D. DNA adduct formation tendency - Xenosite reactivity $\mathbf{2 . 0}$

Xenosite reactivity 2.0 is an online resource for predicting reactivity of any metabolite with DNA thereby identifying which molecule can potentially lead to DNA adduct formation [15], [27], [28]. In xenosite reactivity 2.0 glutathione is considered as an indicator of drug toxicity. Along with DNA it also predicts the reactivity of a molecule with cyanide, GSH and proteins. These predictions range from a score of 0 to 1 and signified by a color shade for the score range as shown in fig. 3 and the site of reactivity is denoted by a circle in the structure of the molecule.

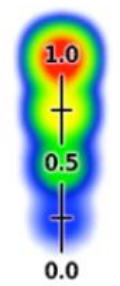

Fig. 3. The reactivity scale of Xenosite reactivity 2.0

III. RESULTS

TABLE 1 - SMARTCYP RESULTS FOR CYP2D6, CYP3A4 AND CYP2C9 ISOFORMS IN CASE OF MODAFIENDZ

\begin{tabular}{|c|c|c|c|c|c|c|}
\hline Rank & Score & $\begin{array}{c}\text { CYP3A4 } \\
\text { Atom number }\end{array}$ & Score & $\begin{array}{c}\text { CYP2D6 atom } \\
\text { number }\end{array}$ & Score & $\begin{array}{c}\text { CYP2C9 atom } \\
\text { number }\end{array}$ \\
\hline 1 & 24.9 & 6 & 56.5 & 6 & 53.3 & 6 \\
\hline 2 & 53.3 & 1 & 61.3 & 1 & 61.3 & 1 \\
\hline 3 & 55.7 & 8 & 86.6 & 8 & 83.4 & 8 \\
\hline
\end{tabular}

TABLE 2 - SMARTCYP RESULTS FOR CYP2D6, CYP3A4 AND CYP2C9 ISOFORMS IN CASE OF MODAFINIL

\begin{tabular}{|c|c|c|c|c|c|c|}
\hline Rank & Score & $\begin{array}{c}\text { CYP3A4 } \\
\text { Atom number }\end{array}$ & Score & CYP2D6 atom number & Score & $\begin{array}{c}\text { CYP2C9 atom } \\
\text { number }\end{array}$ \\
\hline 1 & 24.7 & 14 & 49.8 & 14 & 47.4 & 14 \\
\hline 2 & 55.7 & 7 & 84.9 & 1 & 83.4 & 7 \\
\hline 3 & 69 & 16 & 86.6 & 7 & 84.9 & 7 \\
\hline
\end{tabular}

SMARTCyp results for modafiendz in table 1 show that the $6^{\text {th }}$ atom position in modafiendz is most susceptible to being attacked by CYP2D6, CYP3A4 and CYP2C9 isoforms, another possible $\mathrm{SOM}$ include $1^{\text {st }}$ and $8^{\text {th }}$ atom position. SMARTCyp shows the score for CYP isoforms based on density functional theory(DFT). In case of modafinil the results in table 2 shows that the $14^{\text {th }}$ atom position is most susceptible to being attacked by the given CYP isoforms, other possible SOM includes $1^{\text {st }}$ and $7^{\text {th }}$ atom positions. In SMARTCyp the atom with lowest score has the highest possibility for being the SOM of the given CYP isoform.

TABle 3 - The RANK ANd delta P VAlues of CYP3A4, CYP2D6 AND CYP2C9 ISOFORMSFOR MODAFIENDZ OBTAINED FROM SOMP.

\begin{tabular}{|c|c|c|c|c|c|c|}
\hline Rank & Delta P & CYP3A4 Atom number & Delta P & CYP2D6 atom number & Delta P & CYP2C9 atom number \\
\hline 1 & 0.993 & 6 & 0.707 & 6 & 0.953 & 6 \\
\hline 2 & 0.419 & 1 & 0.663 & 1 & 0.684 & 1 \\
\hline 3 & -0.091 & 2,3 & -0.902 & 3 & -0.916 & 3 \\
\hline
\end{tabular}

TABLE 4 - THE RANK AND DELTA P VALUES OF CYP3A4, CYP2D6 AND CYP2C9 ISOFORMS FOR MODAFINIL OBTAINED FROM SOMP.

\begin{tabular}{|c|c|c|c|c|c|c|}
\hline Rank & Delta P & CYP3A4 Atom number & Delta P & CYP2D6 atom number & Delta P & CYP2C9 atom number \\
\hline 1 & 0.996 & 14 & 0.842 & 14 & 0.977 & 14 \\
\hline 2 & -0.994 & 17 & 0.219 & 1,11 & 0.043 & 1,11 \\
\hline 3 & -0.993 & 18 & 0.146 & $2,6,10,12$ & -0.992 & 17 \\
\hline
\end{tabular}

SOMP results for modafiendz shows the highest rank for $6^{\text {th }}$ atom position in case of CYP2D6, CYP3A4 and CYP2C9. SOMP and SMARTCyp both predict SOM but use different algorithm for that, SMARTCyp uses DFT whereas SOMP uses PASS. The SOMP results for modafinil shows the highest rank for $14^{\text {th }}$ atom position in case of
CYP2D6, CYP3A4 and CYP2C9 as shown in table 3 and 4. In case of SOMP the atom with the highest value of delta $\mathrm{P}$ has the highest probability of being the SOM of the given CYP isoforms.

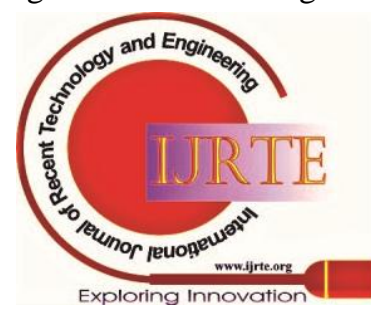


TABLE 5-SOME OF ADME PROPERTIES OF MODAFIENDZ AND MODAFINIL AS PREDICTED BY SWISSADME

\begin{tabular}{|c|c|c|}
\hline Properties & Modafiendz & Modafinil \\
\hline Molecular formula & C16H15F2NO2S & C15H15NO2S \\
\hline Molecular weight & $323.36 \mathrm{G} / \mathrm{mol}$ & $273.35 \mathrm{~g} / \mathrm{mol}$ \\
\hline GI absorption & High & High \\
\hline BBB permeant & Yes & Yes \\
\hline $\begin{array}{c}\text { Lipinski's rule } \\
\text { violations }\end{array}$ & No & No \\
\hline $\begin{array}{c}\text { Ghose's rule } \\
\text { violations }\end{array}$ & No & No \\
\hline $\begin{array}{c}\text { Veber's rule } \\
\text { violations }\end{array}$ & No & No \\
\hline $\begin{array}{c}\text { Egan's rule } \\
\text { violations }\end{array}$ & No & No \\
\hline $\begin{array}{c}\text { Meugge's rule } \\
\text { violations }\end{array}$ & No & 0.55 \\
\hline PAINS formation & 0.55 & 1.92 \\
\hline $\begin{array}{c}\text { Bioavailability } \\
\text { score }\end{array}$ & 2.9 & \\
\hline $\begin{array}{c}\text { Log P o/w } \\
\text { (lipophilicity) }\end{array}$ & & \\
\hline
\end{tabular}

Table 5 shows the ADME properties displayed by Modafiendz and modafinil respectively by SwissADME. As per the result both modafinil and modafiendz follow Lipinski's rule of 5, Ghose's rule, Veber's rule, Egan's rule and Muegge's rule and display no violations.These ruleswere checked for the drug likeness of the molecule. The result also shows that both modafiendz and modafinil have high GI (gastrointestinal) absorption. The bioavailability score of both molecules was also predicted to be the same. Bioavailability is the proportion of the administered drug or other substance that ends up in circulation when is introduced in the body so as to have an active effect. The lipophilicity of modafiendz was predicted to be greater than that of modafinil. Lipophilicity of an ideal drug molecule should not betoo high or too low as the drug properties get adversely affected in these cases [29].Modafiendz and modafinil both was predicted to be blood-brain barrier permeant.

TABle 6 - Possible Chemical Reactions of MODAFIENDZ OBTANIED USING BIOTRANSFORMER

\begin{tabular}{|l|l|c|}
\hline S. no & Metabolite & $\begin{array}{c}\text { Type of reaction } \\
\text { Hydroxylation of } \\
\text { benzene on ortho } \\
\text { carbon to an } \\
\text { electron donating } \\
\text { group }\end{array}$ \\
\hline 2 & $\begin{array}{c}\text { Epoxidation of } \\
\text { arene }\end{array}$ \\
\hline
\end{tabular}

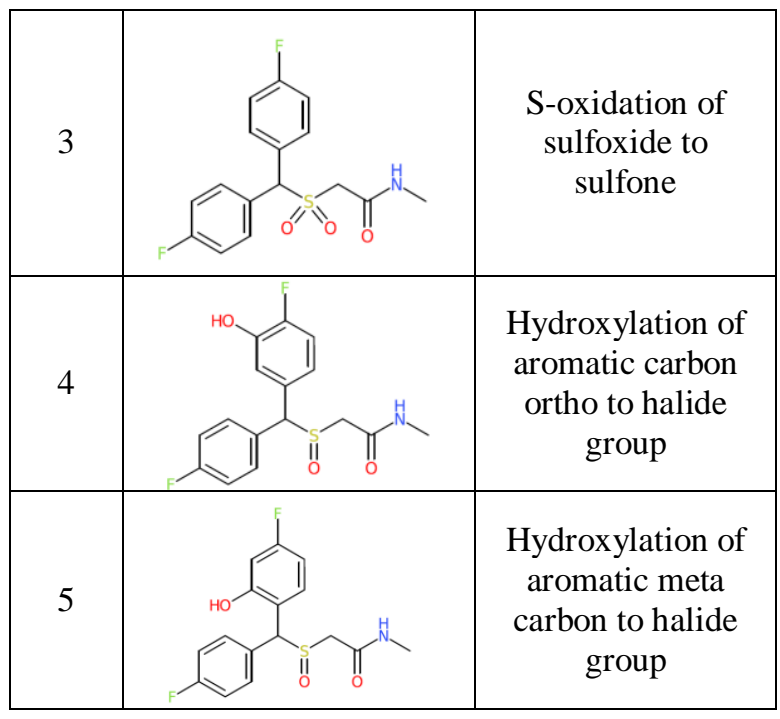

TABLE 7 - POSSIBLE CHEMICAL REACTIONS OF MODAFINIL OBTAINED USING BIOTRANSFORMER

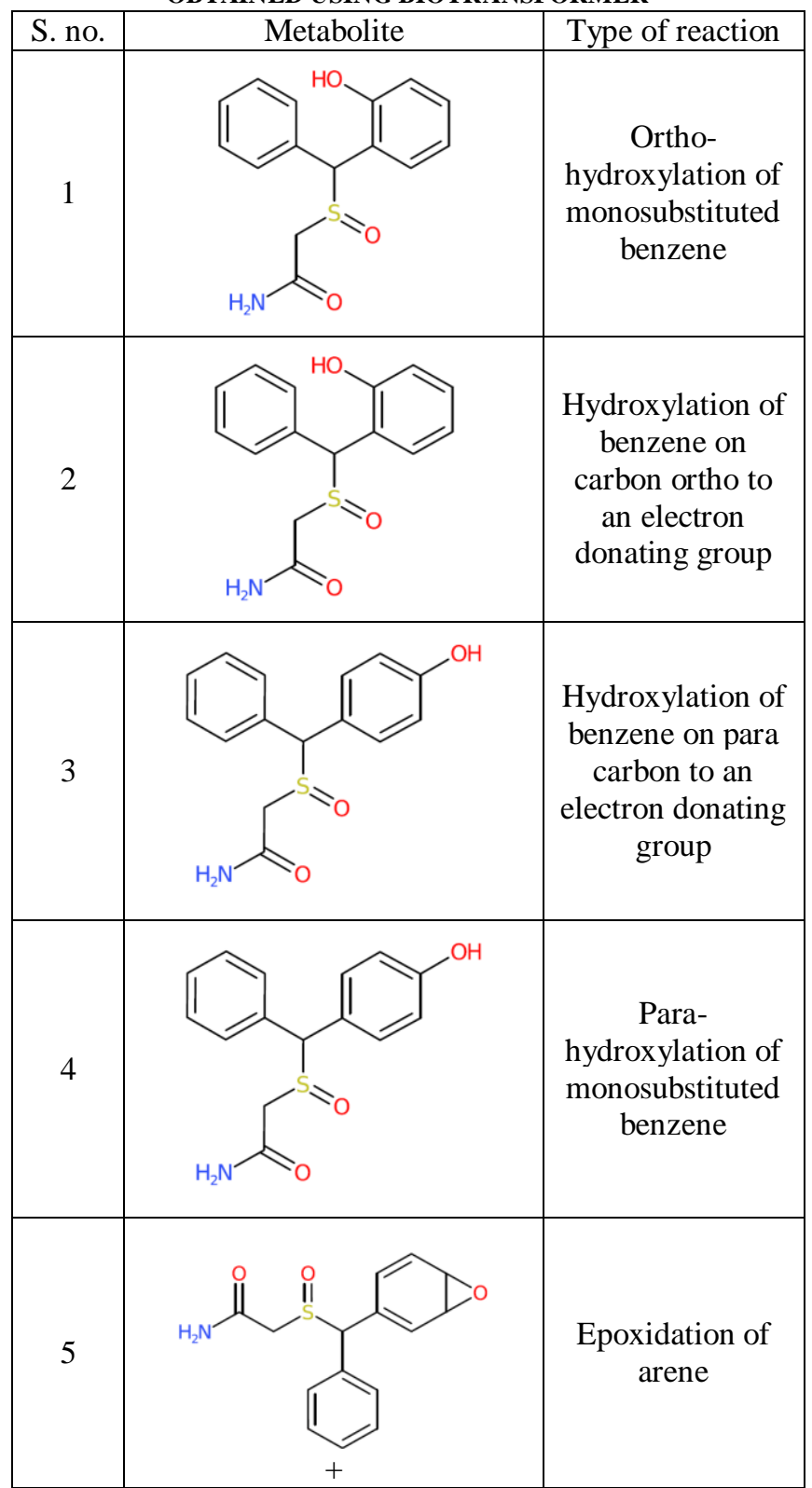

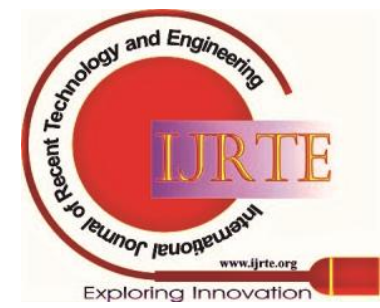




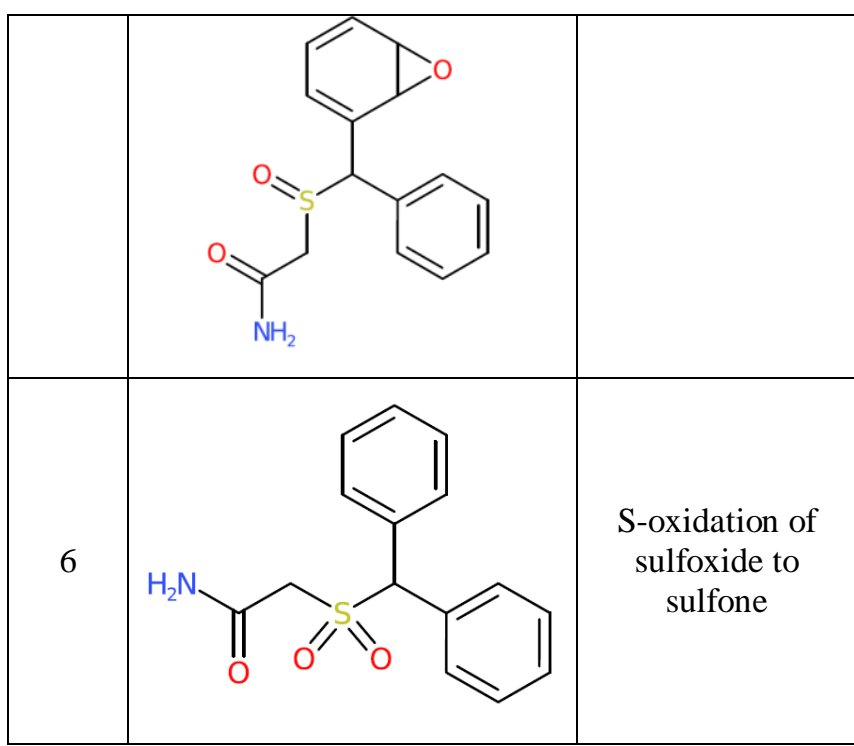

Table 6 and 7 shows the possible reactions that both modafiendz and modafinil can undergo during phase I drug metabolism and the possible resulting metabolites thus obtained using biotransformer tool. The structure of the molecule is used to check with database of chemical reactions and possible biotransformations are deduced. In case of modafiendz 4 unique metabolites are predicted(Table 6) resulting from 5 possible reactions and in case of modafinil 5 unique metabolites are predicted(Table 7) resulting from 6 possible reactions. BioTransformer gives its results based on detailed annotations of experimentally confirmed metabolic reactions while applying preference and transformation rules.

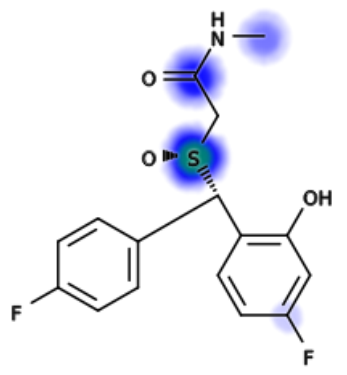

A

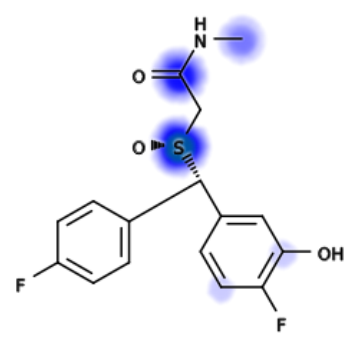

$\mathrm{C}$

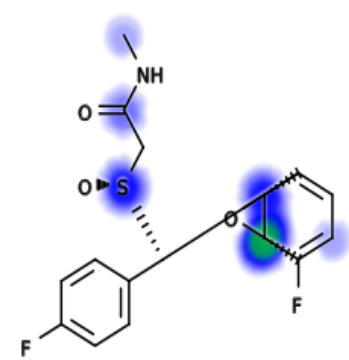

B

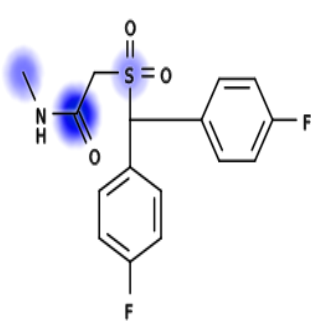

$\mathrm{D}$
Fig. 4. The DNA reactivity diagram of the metabolites of modafiendz predicted using xenosite reactivity $\mathbf{2 . 0}$

$[15,28]$

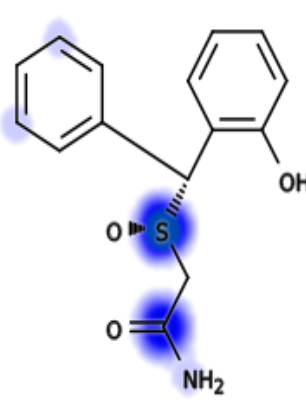

A

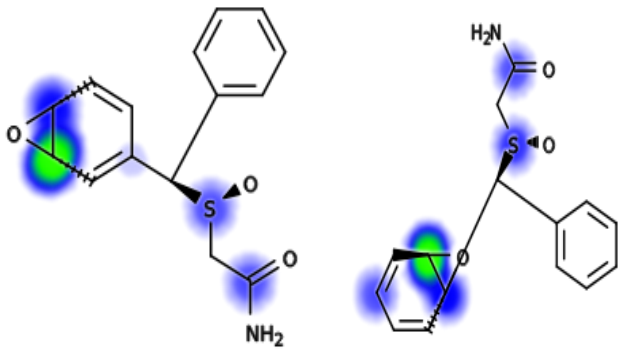

$\mathrm{C}$

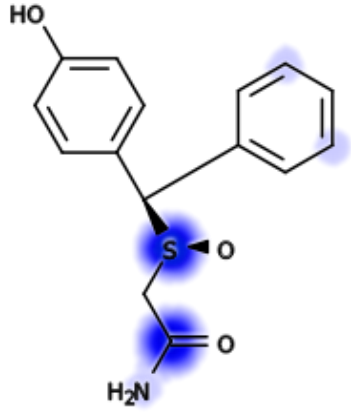

B

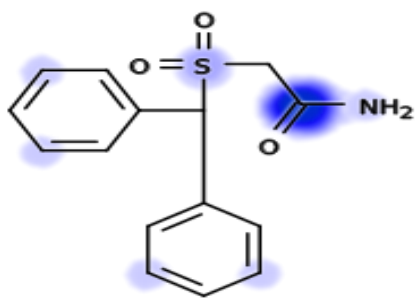

E

Fig. 5. The DNA reactivity diagram of the metabolites of modafinil predicted using xenosite reactivity server 2.0

[15], [28]

Fig. 4 and 5 shows the DNA reactivity figures of the possible metabolites of phase I metabolism of modafiendz and modafinil respectively with the help of predicted using $\begin{array}{llll}\text { xenosite } & \text { reactivity } & 2.0 & \text { serve }\end{array}$ http://swami.wustl.edu/xenosite/p/reactivity [15], [28].

\section{DISCUSSION}

To check the effectiveness of modafiendz as an effective nootropic the properties of modafiendz were deduced using a number ofwell-established softwares in this study. The same procedure was also used for deducing the properties of an earlier known nootropic drug modafinil, the properties of the two compounds were compared as the two molecules are structural analogues. CYP450 enzymes have a major role in metabolism of drugs or xenobiotics in human body.

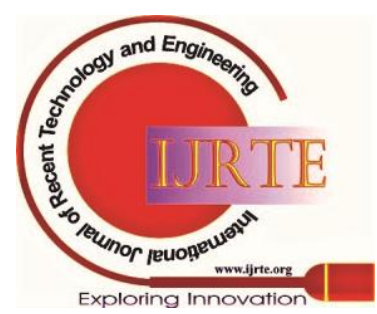


When a xenobiotic enters human body the CYP enzymes act on the molecule and convert it into lesser harmful forms. In this study SMARTCyp and SOMP software were used to find the sites of metabolism. Both the software predict SOM but with different algorithms, SMARTCyp works on the density functional theory(DFT) which determines the activation energies of the SOM and it is used to obtain the final score for a site for being the SOM in given CYP isoforms with lowest score for a site signifying the highest possibility of it being the SOM. SMARTCyp can predict the SOM for 3 CYP isoforms which are most commonly involved in drug metabolism. In case of SOMP the SOM can be obtained by PASS(Prediction of Activity Spectra for Substances) technology and LMNA descriptors. SMARTCyp was first released in 2010 whereas SOMP was released in 2015. These two resources offer two different approaches to find the SOM and hence they were used. The comparison of results of SMARTCyp and SOMP for modafiendz and modafinil is given in Table 8 .

TABLE 8 - COMPARISON OF RESULTS OF SMARTCYP AND SOMP FOR CYP ISOFORMS IN CASE OF MODAFIENDZ AND MODAFINIL.

\begin{tabular}{|c|c|c|c|}
\hline & Rank & SMARTCyp & SOMP \\
\hline \multicolumn{4}{|c|}{ CYP3A4 } \\
\hline \multirow{3}{*}{ Modafiendz } & 1 & 6 & 6 \\
\hline & 2 & 1 & 1 \\
\hline & 3 & 8 & 2,3 \\
\hline \multirow{3}{*}{ Modafinil } & 1 & 14 & 14 \\
\hline & 2 & 7 & 17 \\
\hline & 3 & 16 & 18 \\
\hline \multicolumn{4}{|c|}{ CYP2D6 } \\
\hline \multirow{3}{*}{ Modafiendz } & 1 & 6 & 6 \\
\hline & 2 & 1 & 1 \\
\hline & 3 & 8 & 3 \\
\hline \multirow{3}{*}{ Modafinil } & 1 & 14 & 14 \\
\hline & 2 & 1 & 1,11 \\
\hline & 3 & 7 & $2,6,10,12$ \\
\hline \multicolumn{4}{|c|}{ CYP2C9 } \\
\hline \multirow{3}{*}{ Modafiendz } & 1 & 6 & 6 \\
\hline & 2 & 1 & 1 \\
\hline & 3 & 8 & 3 \\
\hline \multirow{3}{*}{ Modafinil } & 1 & 14 & 14 \\
\hline & 2 & 7 & 1,11 \\
\hline & 3 & 1 & 17 \\
\hline
\end{tabular}

The ADME properties of the two molecules were also calculated using SwissADME software that was released in 2017 and predicts the ADME properties, pharmacokinetic parameters and drug likeliness of a molecule. SwissADME shows the results under physiochemical properties, lipophilicity, solubility, pharmacokinetic properties, medicinal chemistry and drug likenessalong with the bioavailability radar image of the molecule. Lipophilicity is the ability of a chemical compound to dissolve or diffuse in fats, lipids and non-polar solvents [30].

When deciding the overall quality of a potential drug, the role of lipophilicity is of prime importance, the value of lipophilicity of a drug molecule should not be too low and neither should the value be too high as in such cases the metabolism, permeability and solubility of the drug may get affected [29]. The physiochemical properties of a molecule include molecular formula, molecular weight and others. In
SwissADME water solubility is predicted using two topology-based methods and a fragmental method [31]-[33]. The pharmacokinetic properties measured by SwissADME include blood brain barrier permeation, skin permeation, GI (gastrointestinal) absorption and others. The drug likeness of a molecule in SwissADME is checked using various rules of druglikeness, these rules check for the structural properties of a molecule and determine whether the molecule can behave as an ideal drug molecule or not. Bioavailability is also a factor for drug likeliness in a molecule. SwissADME also tells us about aspects of medicinal chemistry like formation of PAINS(pan assay interference compounds) and others. PAINS are compounds that affect the correct results in a number of high throughput screening methods by giving false positives [34]. A comparison of properties of modafiendz and modafinil as predicted by SwissADME is give below in table 2 .

From table 2 it was found that Modafiendz has additional 2 fluorine and 1 carbon atoms when compared to modafinil and is also a heavier molecule than modafinil. Both were predicted to have high gastrointestinal absorption and same amount of bioavailability. Both GI absorption and bioavailability are important parameters of a drug molecule. It is after looking at the bioavailability of a drug molecule that the amount of dose that needs to be administered to a patient can be determined. Both modafiendz and modafinil show no violations of Lipinski's, Ghose's, Veber's, Egan's and Meugge's drug likeability tests. Both molecules were predicted to be blood brain barrier (BBB) permeable. The $\mathrm{BBB}$ is an important selectively permeable membrane which controls what enters the brain and it allows only few smaller molecules to pass through it, most of the drugs are not able to pass through the blood brain barrier [36]. The ability of a drug molecule to pass through blood brain barrier is found to increase with higher level of lipophilicity of the molecule [37].

When analyzed with BioTransformer, modafiendz was predicted to undergo 5 different reactions under phase 1 metabolism, the types of reactions include hydroxylation, epoxidation and $\mathrm{S}$ oxidation and from the reactions 4 unique metabolites were predicted. Modafinil was predicted to undergo 6 different reactions under phase 1 metabolism, the types of reactions include o- and p- hydroxylation (ortho and para), hydroxylation, epoxidation and $\mathrm{S}$ oxidation. From the reactions 5 unique metabolites were predicted. Comparatively modafinil was found to undergo more variety of reactions and produces greater number of metabolites than modafiendz. Out of the reactions predicted for modafiendz and modafinil, 3 were found to be the same i.e S-oxidation of sulfoxide to sulfone, hydroxylation of benzene on carbon ortho to an electron donating group and Epoxidation of arene. The metabolites of modafiendz and modafinil were checked for their DNA binding ability using xenobiotic reactivity 2.0. The color at SOM of molecule depicts the score, where white color signifies nonexistent DNA reactivity;red color signifies maximum reactivity with DNA. In fig 4 from the predicted metabolites of modafiendz 3 (1st and 2nd) of the 4 metabolites showed medium reactivity with DNA(seen in green color) with 2nd metabolite showing the highest score among the other metabolites (signified by its darker shade of green color). In fig 5 from the predicted metabolites of modafinil 2 (3rd

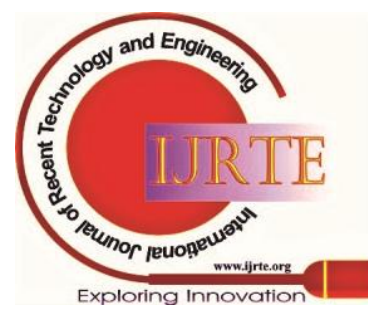


and 4th) of the 5 metabolites showed medium DNA reactivity(signified by green color) and both of the metabolites displayed same score(due to the same shade of green color), however, the shade of green was darker in metabolites of modafinil when compared to metabolites of modafiendz. Hence the metabolites of modafinil have greater tendency to form DNA adducts.

\section{CONCLUSION}

Using the different metabolic pathway prediction tools, the properties and chemical nature of the molecules were predicted. The properties of modafiendzwere found to besimilar to modafinil in various regards of drug likeability, bioavailability,blood brain permeability (BBB), GI absorption andsite of metabolism (SOM). However, the metabolites of modafiendzshowed to have lesser tendency to form DNA adducts and alsopredicted to have BBB permeablity, this makes modafiendz a better nootropic drug candidate, when compared to modafinil.

In coming years, the use of in-silico techniques and software for drug properties analysis and pathway prediction would not only improve but would garner greater attention and application from research world.It is expected that in near future more and more tests and analysis techniques would be freely accessible and available to all. It is because of this easy accessibility and high functionality of these approaches the research work has been made easier and accessible to everyone and hence such platforms promote research and innovation across the globe.

\section{ACKNOWLEDGMENT}

The authors express the deep sense of gratitude to the Department of Biotechnology and Department of Life Sciences, Graphic Era Deemed to be University for all the support,assistance and constant encouragements to carry out this work.

\section{REFERENCES}

1. N. A.Suliman, C. N. M. Taib, M. A.M. Moklas, M. I. Adenan, M. T H. Baharuldin andR.Basir, "Establishing Natural Nootropics: Recent Molecular Enhancement Influenced by Natural Nootropic,"Evid. Based Complement. Alternat. Med., pp. 1-12, 2016.

2. Michael S.Gazzaniga, The Ethical Brain: The Science of Our Moral Dilemmas (P.S.). New York, N.Y: Harper Perennial., 2006, p. 75.

3. C. Giurgea,"Pharmacology of integrative activity of the brain. Attempt at nootropic concept in psychopharmacology," Actual. Pharmacol., 25, pp. 115-116, 1972.

4. Zion Market Research "Global Nootropics Market Growth at 2019. 2024" LP InformationInc, Jan 11, 2019. [Online]. Available” Global News Wire, http://www.lpinformationdata.com/reports/2466/global-

5. G. Norman, "Drugs, Devices, and the FDA: Part 1: An Overview of Approval Processes for Drugs," JACC Basic Transl Sci., 1(3), pp. 170-179,Apr. 2006.

6. NCIthesaurus - modafinil, https://ncit.nci.nih.gov/

7. Provigil - Food and drug administration(FDA), https://www.fda.gov/downloads/drugs/drugsafety/ucm231722.pdf

8. G. Dowling, P. V. Kavanagh, B. Talbot, J. O’Brien, , G. Hessman, , G. McLaughlin andS. D.Brandt, "Outsmarted by nootropics? An investigation into the thermal degradation of modafinil, modafinic acid, adrafinil, CRL-39,940 and CRL-40,941 in the GC injector: formation of $1,1,2,2$-tetraphenylethane and its tetra fluoro analog,” Drug Test Anal., vol 9(3), pp. 518-528, Mar. 2017.

9. M. Billiard and R. Broughton, "Modafinil : its discovery, the early European and North American experience in the treatment of narcolepsy and idiopathic hypersomnia, and its subsequent use in other medical conditions," Sleep medicine, 49, pp. 69-72, 2018. nootropics-market. [Accessed May 10, 2019\}

10. Christoph Wittmann and Sang Yup Lee. Systems metabolic engineering, Springer, 2012, pp. 4-26.

11. S. A.Sheweita,"Drug-metabolizing enzymes mechanisms and functions," Current drug metabolism, 1(2), pp. 107-132, 2000.

12. D. Bryson, P. L. Lim, A. Lawson, A. Lawson, S. Manjunath and G. M. Raner, "Isotopic labelling of the heme cofactor in cytochrome p450 and other heme proteins," Biotechnol. Lett., 33(10), pp. 2019 2026, 2011.

13. J. D. Maréchal, C. A. Kemp, G. C. Roberts, M. J. Paine, C. R. Wolf, and M. J. Sutcliffe, "Insights into drug metabolism by cytochromes P450 from modelling studies of CYP2D6-drug interactions," Br. J. Pharmacol., 153 Suppl 1(Suppl 1), pp. S82-S89, 2007.

14. F. P. Guengerich, "Cytochrome p450 and chemical toxicology" Chem.Res. Toxicol., 21(1), pp. 70-83, 2007.

15. Y. Djoumbou-Feunang, J.Fiamoncini, A. Gil-de-la-Fuente, R.Greiner, C. Manachand D. S. Wishart, "BioTransformer: a comprehensive computational tool for small molecule metabolism prediction and metabolite identification," J. cheminform., 11(1), pp. 1-25, 2019.

16. T. B. Hughes, N. L. Dang, G. P. Millerand and S. J. Swamidass, "Modeling reactivity to biological macromolecules with a deep multitask network," ACS Cent. Sci., 2(8), pp. 529-537, 2016

17. P. P.Massion, andD. P. Carbone, "The molecular basis of lung cancer: molecular abnormalities and therapeutic implications," Respir. Res., 4(1), p. 12, 2003.

18. Patrik Rydberg, David E. Gloriam and Lars Olsen, "The SMARTCyp cytochrome P450 metabolism prediction server," Bioinformatics, Volume 26, Issue 23, pp. 2988-2989, 1 Dec. 2010.

19. S. Jamuna, A. Rathinavel, S. S. Mohammed Sadullah, and S. N. Devaraj, "In silico approach to study the metabolism and biological activities of oligomeric proanthocyanidin complexes," Indian J.Pharmacol., 50(5), pp. 242-250, 2018.

20. J. Kirchmair, M. J. Williamson, J. D. Tyzack, L.Tan, P. J.Bond, A Bender and R. C. Glen, "Computational prediction of metabolism: sites, products, SAR, P450 enzyme dynamics, and mechanisms," J. Chem. Inf. Model., 52(3), pp. 617-648, 2012.

21. A.Rudik, A. Dmitriev, A.Lagunin, D. Filimonov and V. Poroikov, "SOMP: web-service for in silico prediction of sites of metabolism for drug-like compounds," Bioinformatics, 31 (12), pp. 2046-2048, 2015.

22. A. Daina, O. Michielin and V. Zoete, "SwissADME: a free web tool to evaluate pharmacokinetics, drug-likeness and medicinal chemistry friendliness of small molecules," Sci. Rep., 7, 42717, 2017 [Online] Available: https://www.nature.com/articles/srep42717

23. C.A. Lipinski, F. Lombardo, B. W. Dominy and P. J. Feeney, "Experimental and computational approaches to estimate solubility and permeability in drug discovery and development settings," Adv. Drug Deliv. Rev., 46, pp. 3-26, 2001.

24. A. K. Ghose, V. N. Viswanadhan and J. J. Wendoloski, “A knowledge-based approach in designing combinatorial or medicinal chemistry libraries for drug discovery. 1. A qualitative and quantitative characterization of known drug databases," J. Comb. Chem., 1(1), pp. 55-68, 1999.

25. D. F. Veber, S. R. Johnson, H. Y. Cheng, B. R. Smith, K. W. Ward, and K. D. Kopple, "Molecular properties that influence the oral bioavailability of drug candidates," J. Med. Chem., 45(12), pp. 2615 2623, 2002.

26. W. J. Egan, K. M. Merz and J. J. Baldwin, "Prediction of drug absorption using multivariate statistics," J. Med. Chem., 43(21), pp. 3867-3877, 2000.

27. I. Muegge, S. L. Heald and D. Brittelli, "Simple selection criteria for drug-like chemical matter," J. Med. Chem., 44(12), pp. 1841-1846, 2001.

28. V. Delannée, S. Langouët, A. Siegel and N. Theret, "In silico prediction of Heterocyclic Aromatic Amines metabolism susceptible to form DNA adducts in humans," Toxico. Lett., 300, pp. 18-30, 2019.

29. T. B. Hughes, G. P. Miller and S. J. Swamidass, "Site of reactivity models predict molecular reactivity of diverse chemicals withglutathione," Chem. Res. Toxicol., 28(4), pp. 797-809, 2015

30. M. J. Waring, "Lipophilicity in drug discovery," Expert Opinion on Drug Discovery, 5(3), pp. 235-248, 2010.

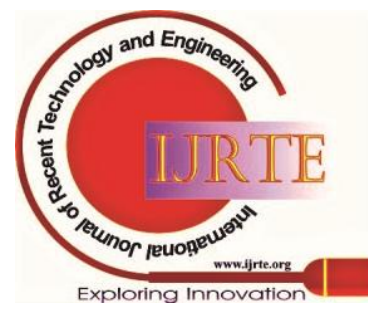


31. A.D. Mcnaught and A. Wilkinson, IUPAC Compendium of Chemical Terminology, 2nd ed, Oxford, 1997.

32. J. S. Delaney, "ESOL: estimating aqueous solubility directly from molecular structure," J. Chem. Inf. Comput. Sci., 44(3), pp. 1000 1005, 2004.

33. S. Kotta, A. W. Khan, K. Pramod, S. H. Ansari, R. K. Sharma and J. Ali, "Exploring oral nanoemulsions for bioavailability enhancement of poorly water-soluble drugs," Expert Opin. Drug Deliv., 9(5), pp. 585-598, 2012.

34. J. L. Dahlin, J. W. M. Nissink, J. M. Strasser, S. Francis, L. Higgins, H. Zhou, and M. A. Walters, "PAINS in the assay: chemical mechanisms of assay interference and promiscuous enzymatic inhibition observed during a sulfhydryl-scavenging HTS," J. Med. Chem., 58(5), pp. 2091-2113, 2015.

35. J. Baell and M. A. Walters, "Chemistry: Chemical con artists foil drug discovery," Nature News, 513(7519), pp. 481-483, 2014.

36. W. M. Pardridge, "The blood-brain barrier: bottleneck in brain drug development," NeuroRx, 2(1), pp. 3-14, 2005.

37. J. A. Arnott and S. L. Planey, "The influence of lipophilicity in drug discovery and design," Expert opinion on drug discovery, 7(10), pp. $863-875,2012$. 